European Conference on Laboratory Astrophysics - ECLA

C. Stehlé, C. Joblin and L. d'Hendecourt (eds)

EAS Publications Series, 58 (2012) 117-125

www.eas.org

\title{
JETS FROM YOUNG STARS AND Z-PINCH MACHINES
}

\author{
A. $\operatorname{Ciardi}^{1}$
}

\begin{abstract}
Outflows and jets are intimately related to the formation of stars, and play a central role in redistributing mass, energy and angular momentum within the core, disk and parent cloud. The interplay between magnetic field and rotation is widely thought to be responsible for launching and collimating these outflows. Shear induced by differential rotation along initially poloidal field lines results in an azimuthal component of the magnetic field being generated; the magnetic pressure gradient then accelerates the plasma, and inflates bipolar magnetic cavities within the circumstellar matter. However, the resulting winding of the magnetic field can be potentially disrupted by magnetohydrodynamic instabilities. To better understand the role of magnetic fields in shaping these ouflows, a series of experiments on pulsed-power z-pinch machines have been developed. In this talk I will present results related to the formation of jets in young stellar objects and in the laboratory, and draw a parallel between the two systems.
\end{abstract}

\section{Introduction}

Although jets and outflows are associated with widely diverse astrophysical environments, they exhibit many common features independent of the central source. For young stellar systems, the existence of a close relation between the ejection and accretion of matter throughout the low-mass $\left(M_{\star} \lesssim 2 M_{\odot}\right)$ star formation history (Cabrit \& Bertout 1992) suggests the presence of a single launching mechanism, which relies on a large-scale magnetic field mediating the extraction of gravitational energy from the accreting plasma, and redirecting mass and energy, in the form of kinetic and Poynting flux, into bi-polar jets. The fundamental magnetohydrodynamic (MHD) mechanism for launching and collimating an axisymmetric, self-similar outflow was detailed in analytical work by Blandford \& Payne (1982) and applied to young stellar systems by Pudritz \& Norman (1983); it was later extended to include non-self similar solutions by Pelletier \& Pudritz (1992) and

${ }^{1}$ LERMA, Observatoire de Paris, ENS, UPMC, CNRS, 5 place J. Janssen, 92195 Meudon, France

(C) The Author(s) 2013

DOI: $10.1051 /$ eas/1258019 
global disc-jet solutions by Ferreira (1997). The basic ideas underlying MHD jet launching were confirmed in time-dependent two-dimensional, axisymmetric numerical simulations (see for example Zanni et al. 2007). Furthermore, the early ejection of bipolar outflows was also demonstrated in two- (Mellon \& Li 2008) and three-dimensional MHD simulations of the collapse of pre-stellar dense cores (Ciardi \& Hennebelle 2010; Hennebelle \& Fromang 2008), which notably included the second collapse and the formation of jets from the protostar (e.g. Machida et al. 2006).

In addition to these "traditional" studies of astrophysics outflows, high-energy density plasma experiments can also address the physics of compressible, radiative, MHD flows (Ciardi et al. 2007, 2009; Lebedev et al. 2005; Suzuki-Vidal et al. 2010). In what follows I will present results related to numerical simulations of outflows and jets produced during the early collapse of pre-stellar dense cores, as well as related work on magnetized laboratory jets.

\section{Scaling}

Astrophysical jets and outflows are described to first approximation by ideal MHD and the laboratory experiments are designed to produce flows in that regime. Its applicability requires the dimensionless Reynolds $(\mathrm{Re})$, magnetic Reynolds $\left(\operatorname{Re}_{m}\right)$ and Peclet $(\mathrm{Pe})$ numbers to be much larger than unity, implying that the transport of momentum, magnetic fields and thermal energy respectively, takes place predominantly through advection with the flow (see Bouquet et al. 2010; Falize et al. 2011; Ryutov et al. 2001, for these scaling laws)

At this point it is important to stress that astrophysical jets have typical values of $\operatorname{Re}>10^{8}, \operatorname{Re}_{m}>10^{15}$ and $\mathrm{Pe}>10^{7}$, that are many orders of magnitude greater than those achieved not only in the laboratory, but also in numerical simulations, which have been so far the sole means of investigating time-dependent behaviour of multi-dimensional MHD jets. In ideal MHD simulations unphysical dissipation occurs at the grid level through numerical truncation errors, and for global jet models, mostly performed assuming axisymmetry, the effective numerical Reynolds numbers are typically in the range $10-10^{3}$. Severe limitations also exist on the range of plasma- $\beta$, the ratio of thermal to magnetic pressure, which may be reliably modelled numerically. The study of astrophysical jets and outflows is further hampered by their great complexity, which requires a full 3D MHD treatment to accurately explicate relevant processes and dynamics. The (inherently) three-dimensional, scaled experiments can extend the range of the dimensionless parameters obtained in the global modelling of jets and represent a complementary new tool to support the study of these astrophysical phenomena. Typical values obtained in the experiments are $\operatorname{Re} \sim 5 \times\left(10^{5}-10^{6}\right), \operatorname{Re} \sim 150-500$ and $\mathrm{Pe} \sim 20-50$ (Lebedev et al. 2005; Ciardi et al. 2009). Finally, similar to astrophysical jets, the flows produced are high Mach number, both sonic and Alfvenic, and are radiatively cooled. The latter is characterized by the cooling parameter $\chi_{\text {cool }}=\tau_{\text {cool }} / \tau_{\text {dyn }}$, where $\tau_{\text {cool }}$ is the characteristic cooling time and $\tau_{d y n}$ is the 
flow dynamical time-scale. Both laboratory and astrophysical jets are characterized by $\chi<1$, and radiation plays an important role in structuring the flow.

\section{Jets in space and in the laboratory}

\subsection{Outflows from collapsing prestellar cores}

In general the formation of bipolar outflow begins as the magnetic field lines, connecting the outer regions of the cloud to its rapidly rotating inner regions, undergo strong shear, which results in a significant azimuthal component $\left(B_{\phi}\right)$ of the magnetic field being generated close to the adiabatic core and disc (if present). The growing magnetic pressure gradient accelerates the plasma, inflating bi-polar magnetic cavities within the infalling envelope. Such "magnetic tower" structure is similar to that described by Lynden-Bell (2003) and re-produced in scaled laboratory experiments (Lebedev et al. 2005), whose simulations are described in the next section. We stress that at this stage of the evolution, the outflow cavity is inflated by the magnetic field, and it is not mechanically-driven by wide-angle winds. In addition, at the base of the cavity, the continuous generation of $B_{\phi}$ provides the Poynting flux powering an outflow which originates from the core and a region extending several AU around it. Depending on the ejection efficiency, this outflow may be described as either magneto-centrifugally or magnetic pressure driven (Ferreira \& Pelletier 1995). The ejection efficiency increases in time and both launching regimes are attained during the simulations.

The collimation of this disc wind into a jet depends on the radial distribution of currents, and therefore on the width (and shape) of the magnetic cavity itself, whose walls carry the return current. In turn, the collimation of the magnetic cavity depends on the distribution of gas and magnetic field in the circumstellar plasma. As a consequence, the medium through which the magnetic cavity expands plays an important role on the overall collimation of the outflow. Figure 1 shows the outflows' three-dimensional structure for different $\alpha$, the initial angle between the magnetic field and the prestellar core rotation axis. Increasing the misalignment leads to the narrowing and better collimation of the whole magnetic cavity (Ciardi \& Hennebelle 2010). The spatial variability is mainly the consequence of growing non-axisymmetric current-driven modes and the presence of episodic ejections. The unsteady behaviour appears to be related to the increasing mass ejection efficiency, and build up of $B_{\phi}$ in the core/disc (Anderson et al. 2005). The precession present in the misaligned models further helps to seed large kink-like perturbations in the jet body, promoting the growth of the instability,which is otherwise artificially suppressed in the aligned case. The presence of a kink-like instability is indeed expected in jets confined by a helical magnetic field, and in detailed three-dimensional simulations it was also seen to lead to considerable distortions of the jet body and its fragmentation (Nakamura \& Meier 2004; Moll et al. 2008). In the simulations we find that the outflows are above the Kruskal-Shafranov stability threshold, with typical values of the magnetic pitch evaluated along single field lines, $\varpi\left|B_{Z}\right| /\left|B_{\phi}\right| \lesssim 30 \mathrm{AU}$ (e.g. Appl et al. 2000), 

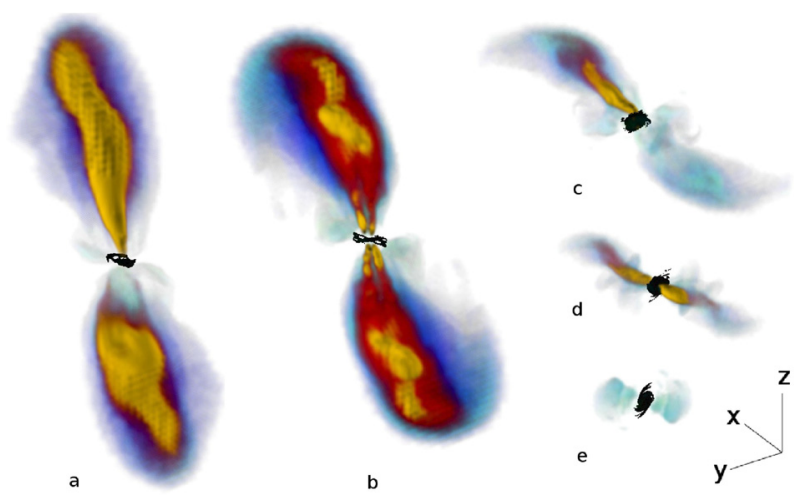

Fig. 1. Volume rendering of the outflows for an angle $\alpha$, between the rotation axis and magnetic field, equal to (a) $20^{\circ}$, (b) $45^{\circ}$, (c) $70^{\circ}$, (d) $80^{\circ}$ and (e) $90^{\circ}$. The colours represent flow regions with increasing speeds (from blue to yellow). The black disc-like regions represent densities $>10^{10} \mathrm{~cm}^{-3}$ (Ciardi \& Hennebelle 2010).

where $\varpi$ is the cylindrical radius. Precession and instabilities therefore lead to the fragmentation of the outflow into clumps, which together with the generation of episodic ejections, produce a series of nested cavities and internal shocks, where faster ejections plough through previously launched material. The scaled laboratory astrophysics experiments of compressible, super-magnetosonic MHD flows presented next, also show that although jets may be episodic and unstable, multiple ejections of magnetic cavities and jets can produce clumpy flows which remain well collimated (Ciardi et al. 2009).

\subsection{Jets in the laboratory}

A schematic diagram of the experimental jet/outflow produced in radial wirearrays is shown in Figure 2. Two outflow components are generally present: a magnetic bubble (or cavity) accelerated by gradients of the magnetic pressure and surrounded by a shell of swept up ambient material, and a magnetically collimated jet on the interior of the bubble. As discussed in the previous section, the confinement of the magnetic cavity itself relies on the presence of an external medium, while the jet is magnetically confined. Episodic jet ejections can be produced in the laboratory by replacing the wires with a foil (Ciardi et al. 2009; Suzuki-Vidal et al. 2010). The dynamics essentially consists of repeated formation and ejection of magnetic cavities and jets as in radial wire arrays. Figure 3 presents simulations of episodic jet ejections obtained by artificially injecting mass in the gap produced between the central electrode and the foil. As the mass fills this gap, the Poynting flux powering the cavity is halted. In the experiments the mass is likely to be generated by the ablation of the electrodes. The growth of large amplitude kink-like perturbations and further expansion of the cavity ultimately lead to the detachment of the jet and the break-up of the cavity, however, a clumpy jet still 

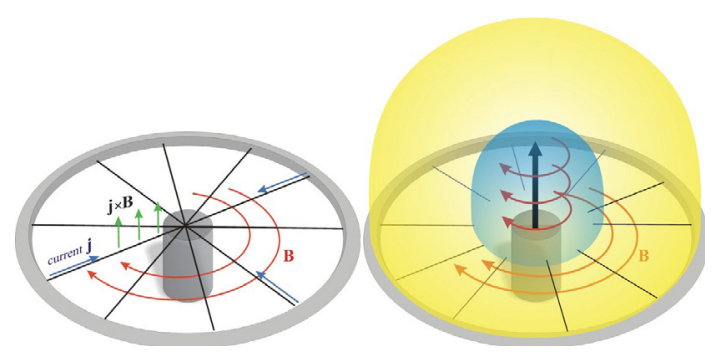

Fig. 2. Schematic of a radial wire array. The results discussed here are based on experiments performed on the MAGPIE Z Pinch at Imperial College (Lebedev et al. 2005).

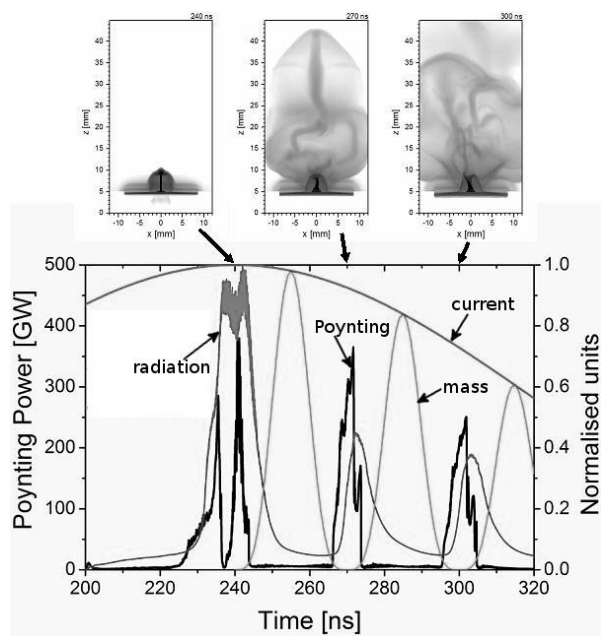

Fig. 3. Episodic cavity/jet ejections. The contour plots show the line-of-sight integrated self-emission, showing the early moment of the jet/cavity ejection.

emerges. The combination of instabilities and episodic ejections leads naturally to a heterogeneous flow able to transport in a narrow channel mass and energy away from the formation region.

\subsubsection{Instabilities in jets}

The jet configuration, a current-carrying column of plasma confined by magnetic field, is known to be prone to potentially disruptive, magnetohydrodynamic instabilities. Depending on the driving mechanism these are broadly classified as current or pressure driven (see Freidberg 1982 for a review). Current-driven instabilities, the so-called kink mode being the most disruptive for jets, are either internal or surface modes which lead to helical $(|m|=1)$ or higher order distortions of the jet, where $m$ is the azimuthal mode number. Linear analysis for idealized jet 
configurations has been performed by Appl et al. (2000). For force-free equilibria it was shown that in the small pitch limit, $P=r B_{z} / B_{\phi}<1$, which corresponds to a dominant azimutal magnetic field, the growth rates are largely independent of the pitch profile. The $|m|=1$ mode is the fastest growing and involves a range of axial wavenumbers, the fastest growing being $k_{z} \sim-m / P$. Pressure-driven instabilities become more important for jets with subthermal fields, where pressure gradients can play a destabilizing role. In general, current and pressure driven modes can coexist, as well as with shear-flow driven instabilities (Baty \& Keppens 2002). The linear stability of jets for the case of subthermal fields, to pressure and current driven instabilities, has shown that contrary to the suprathermal case, stabilization never occurs. Even in the limit of relatively large pitch values, $B_{z} \gtrsim B_{\phi}$, the higher order azimuthal modes $|m| \gg 1$ are unstable and have the largest growth rates (Bonanno \& Urpin 2011). In general, linear theory predicts growth rates of the order of the inverse Alfven crossing time, which can represent growth times significantly shorter than the jet evolution time-scale. Few three-dimensional simulations have addressed the stability of more realistic, essentially non-equilibrium, jet configurations. The results show the existence of possible stabilization mechanisms however the long-term stability of jets remains unsettled (Nakamura \& Meier 2004; Moll et al. 2008; Huarte-Espinosa et al. 2012) Going back to laboratory jets, the growth time of the $|m|=1$ modes was estimated to be of the order of a few nanoseconds (Lebedev et al. 2005), much shorter than the characteristic jet evolution time and, as for astrophysical jets, it is of the order of the Alfven crossing time. In addition, radiative cooling effects can increase the growth rates. Figure 4 shows the line-of-sight integrated current density for a Tungsten jet, and a similar simulation where the cooling has been artificially suppressed. Although idealized, the simulation shows the persistence of the "stable" jet for a longer time. Indeed, the power spectrum for the $|m|=1$ mode, shows that in the linear regime, the growth of perturbations with no radiation losses is slower. This behaviour can be understood in simple terms by considering that the growth rate is approximately $\gamma \sim v_{\phi A} / R_{j}$, where $v_{\phi A}$ is the toroidal Alfven speed and $R_{j}$ is the jet radius. Assuming the current and total mass in the jet are the same for the two cases, gives $v_{\phi A} \sim$ const, and a growth rate that scales as $\gamma \propto 1 / R_{j}$. Radiation losses tend to reduce the thermal pressure of the plasma and thus the equilibrium radius of the jet, leading to larger growth rates. However changing the wire material, which modifies the cooling rates, may not necessarily provide a means of testing this effect. Materials of lower atomic numbers will produce jets with larger ion line densities, and thus complicate the simple picture given above.

\subsubsection{Phenomenological scaling}

One can gain useful insights and a qualitative view of the possible evolution of astrophysical jets by drawing a parallel with the dynamics observed in the experiments. In the episodic jet experiments there are two basic time-scales of interest that can be related to astrophysical jets. The first is the growth time of the instabilities, which is a few nanoseconds in the experiments, and that for conditions 

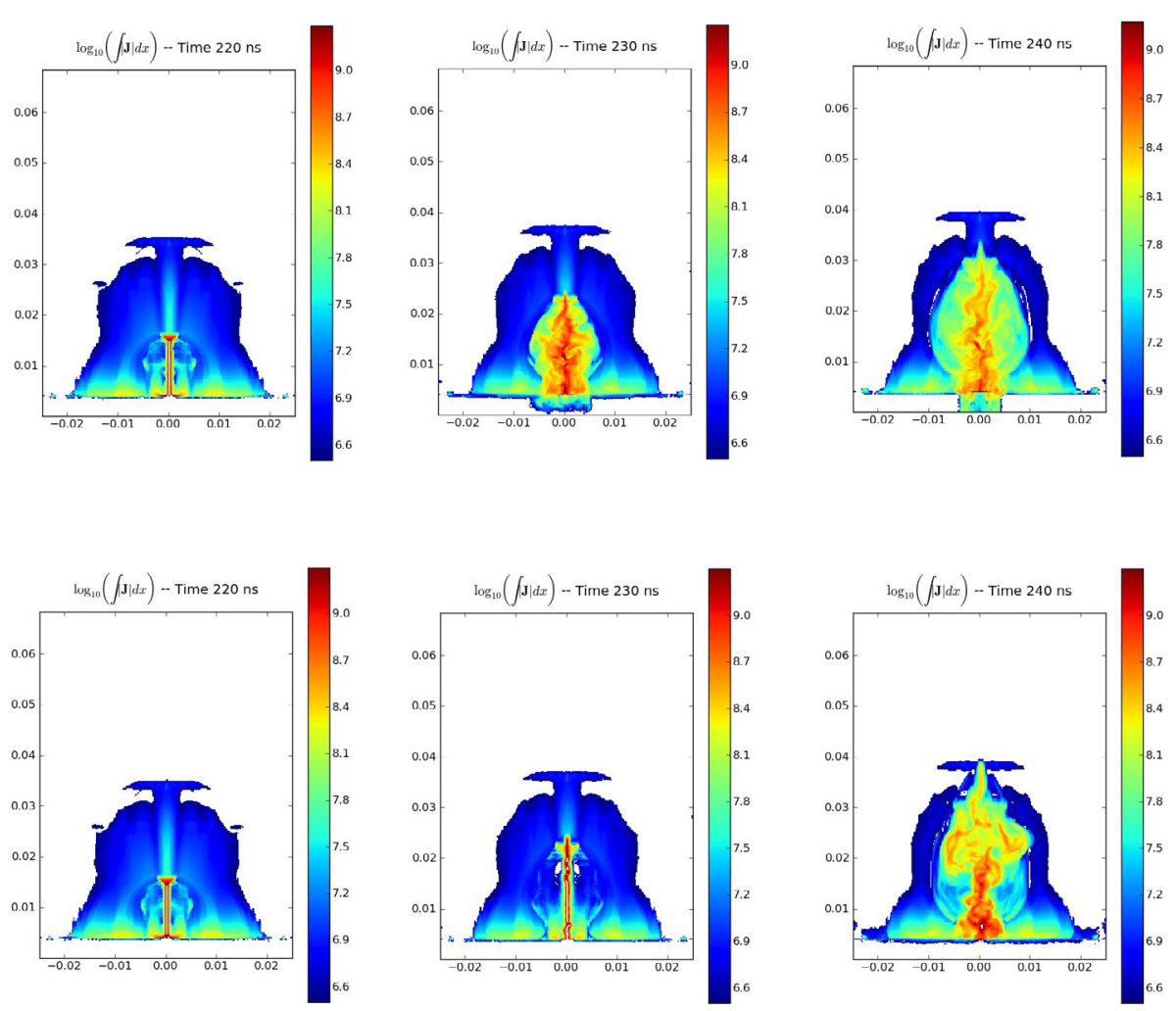

Fig. 4. Line-of-sight integrated current density for a Tungsten radial wire array (top) and an equivalent simulation with the radiative cooling artificially suppressed.

applicable to the formation region of protostellar jets (Hartigan et al. 2007) can be estimated as the Alfven crossing time, which is of the order of $\sim 1$ year. The second time-scale is the relatively longer bubble ejection period, linked to the temporal variability of the Poynting flux feeding the bubbles. For astrophysical sources it will be associated with a substantial variation in the outflow launching activity. Although the mechanism responsible is unknown, observations of knots kinematics, assumed to trace ejection variability, suggest characteristic times of several years, and we take the bubble ejection period to be of the same order. We stress that both time-scales are relatively longer than the characteristic Keplerian period of rotation at the inner disk radius, and in this respect jet launching should have ample time to reach steady-state. By using scalings given in Table 1, we can draw a parallel between protostellar outflows and the dynamics observed in the experiments, as shown in Figure 5. The presence of multiple bubble-like features should be observed on scales ranging from a few tens to a few hundred AU from the source. Because of the relatively short growth time of the instabilities, jets should develop non-axisymmetric features already within a few tens AU from the 


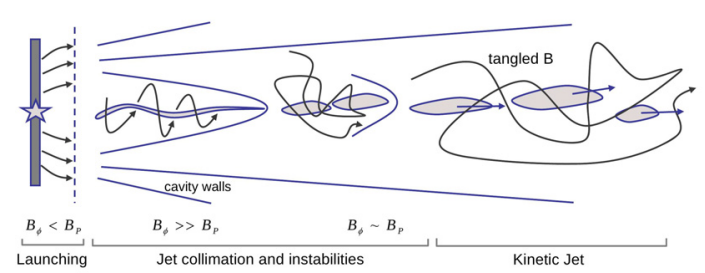

Fig. 5. Schematic model of a stellar jet based on scaling up the observed laboratory dynamics.

Table 1. Characteristic scaling parameters.

\begin{tabular}{|ccc|}
\hline & Astro & Lab \\
$v$ & $10^{7} \mathrm{~cm} / \mathrm{s}$ & $10^{7} \mathrm{~cm} / \mathrm{s}$ \\
$n$ & $10^{6} \mathrm{~cm}^{-3}$ & $10^{19} \mathrm{~cm}^{-3}$ \\
$t$ & 1 year & $10 \mathrm{~ns}$ \\
$x$ & $21 \mathrm{AU}$ & $1 \mathrm{~mm}$ \\
$p$ & $10^{-10}$ & $16 \mathrm{kbar}$ \\
$B$ & $1 \mathrm{mG}$ & $1 \mathrm{~T}$ \\
$\chi_{\text {cool }}$ & $<1$ & $<1$ \\
\hline
\end{tabular}

source, and become more heterogeneous and clumpy as they move further away to hundreds of AU. Over the same length scales we would expect magnetic energy dissipation, heating of the plasma and a transition to a kinetically dominated jet which propagates ballistically. Compelling similarities with observation of the young stellar system DG Tau are discussed by Agra-Amboage et al. (2011).

I would like to thank the organizers for the invitation to this new conference and the collaborators to the work presented here: S.V. Lebedev, F. Suzuki-Vidal, A. Frank and P. Hennebelle. Some computations were performed at the Barcelona Supercomputing Centre through the HPCEUROPA2 program. I acknowledge financial support from "Programme National de Physique Stellaire" of CNRS/INSU.

\section{References}

Agra-Amboage, V., Dougados, C., Cabrit, S., \& Reunanen, J., 2011, A\&A, 532, A59

Anderson, J.M., et al., 2005, ApJ, 630, 945

Appl, S., et al., 2000, A\&A, 355, 818

Baty, H., \& Keppens, R., 2002, ApJ, 580, 800

Blandford, R.D., \& Payne, D.G., 1982, MNRAS, 199, 883

Bonanno, A., \& Urpin, V., 2011, A\&A, 525, A100

Bouquet S., Falize E., Michaut C., et al., 2010, HEDP, 6, 368

Cabrit, S., \& Bertout, C., 1992, A\&A, 261, 274

Ciardi, A., et al., 2007, PhPl, 14, 056501 
Ciardi, A., et al., 2009, ApJ, 691, L147

Ciardi, A., \& Hennebelle, P., 2010, MNRAS, 409, L39

Falize, É., Michaut, C., \& Bouquet, S., 2011, ApJ, 730, 96

Ferreira, J., \& Pelletier, G., 1995, A\&A, 295, 807

Ferreira, J., 1997, A\&A, 319, 340

Freidberg, J.P., 1982, Rev. Mod. Phys., 54, 801

Hartigan, P., et al., 2007, ApJ, 661, 910

Hennebelle, P., \& Fromang, S., 2008, A\&A, 477, 9

Hennebelle, P., \& Ciardi, A., 2009, A\&A, 506, L29

Huarte-Espinosa, et al., 2012

Lebedev, S.V., et al., 2005, MNRAS, 361, 97

Lynden-Bell, D., 2003, MNRAS, 341, 1360

Machida, M.N., et al., 2006, ApJ, 647, L1

Mellon, R.R., \& Li, Z., 2008, ApJ, 681, 1356

Moll, R., et al., 2008, A\&A, 492, 621

Nakamura, M., \& Meier, D.L., ApJ, 617, 123

Pelletier, G., \& Pudritz, R.E., 1992, ApJ, 394, 117

Pudritz, R.E., \& Norman, C.A., 1983, ApJ, 274, 677

Ryutov, D.D., Remington, B.A., Robey, H.F., \& Drake, R.P., 2001, PhPl, 8, 1804

Suzuki-Vidal, F., et al., 2010, PhPl, 17, 112708

Zanni, C., et al., 2007, A\&A, 469, 811 